\title{
Armut bekämpfen
}

\section{Die Nationale Armutskonferenz in Deutschland ist Teil des Europäischen Armutsnetzwerkes}

\author{
Michael Schröter
}

Michael Schröter ist im Zentrum Familie, Integration, Bildung, Armut des Diakonischen Werkes der EKD e. V. in Berlin für die Geschäftsführung der Nationalen Armutskonferenz zuständig. E-Mail schroeter@diakonie.de
Die Nationale Armutskonferenz ist ein Zusammenschluss der Spitzenverbände der Freien Woblfahrtspflege, bundesweit tätiger Fachverbände und Selbsthilfeorganisationen und des Deutschen Gewerkschaftsbundes. Die Konferenz gründete sich im Herbst 1991 als deutsche Sektion des Europäischen Armutsnetzwerkes.

Nach allgemeinem Verständnis gilt als arm, wer sich nicht hinreichend und angemessen mit Kleidung, Lebensmitteln, Wohnraum und anderen lebensnotwendigen Dingen versorgen kann. Die Europäische Union hat die relative Armut im Jahre 1984 wie folgt beschriebenen: "Als verarmt sind jene Einzelpersonen, Familien und Personengruppen anzusehen, die über so geringe (materielle, kulturelle und soziale) Mittel verfügen, dass sie von der Lebensweise ausgeschlossen sind, die in dem Mitgliedstaat, in dem sie leben, als Minimum annehmbar sind.

In Deutschland wird das durchschnittliche, gewichtete monatliche Netto-Einkommen als wichtigster Indikator zugrunde gelegt; dies bedeutet, dass auch die Anzahl der Personen im jeweiligen Haushalt berücksichtigt wird. Wer weniger als 60 Prozent davon zur Verfügung hat, gilt als arm. Im Jahr 2008 lag die Armutsrisikogrenze für einen Einpersonenhaushalt bei 787 Euro monatlich, für einen Zweipersonenhaushalt betrug sie 1.181 Euro und für einen Vierpersonenhaushalt (Eltern, zwei Kinder unter 14 Jahren) betrug sie 1.653 Euro (Datenbasis Mikrozensus).

Armut hat viele Gesichter. Besonders betroffen sind drei Millionen Kinder, 42 Prozent der Alleinerziehenden, Arbeitslose, ältere Menschen, Behinderte und soziale Randgruppen. 1,31 Millionen Menschen beziehen trotz Erwerbstätigkeit ergänzend Leistungen nach dem SGB II. Kaum zu beziffern ist das Ausmaß der »verdeckten Armut « von Menschen, die meist aus Scham keine öffentliche Hilfe in Anspruch nehmen.
Vor diesem Hintergrund lässt sich feststellen, dass Armut nicht allein ein Problem von Erwerbslosigkeit darstellt. Vielmehr ist in Deutschland eine umfassende soziale Grundsicherungsleistung nötig, die weitere Lebensrisiken verlässlich absichert. Ergänzend müssen Maßnahmen umgesetzt werden, die sicher stellen, dass Erwerbseinkommen zum Leben reicht.

Um eine umfassende Beschreibung und Bekämpfung von Armut zu ermöglichen, muss die Armuts- und Reichtumsberichterstattung in Deutschland fortgeschrieben werden. Ansonsten wird Armut verdeckt und gerät aus dem Blickfeld. Armut und Ausgrenzung überwinden heißt, in Gerechtigkeit zu investieren und soziale Ungleichheit zu vermindern. Hierzu gehört einerseits ein Leistungssatz für die soziale Grundsicherung, der die tatsächlichen Bedarfe abbildet. Andererseits sind umfassende soziale Hilfen wie u. a. Sicherung von Wohnraum, umfassende persönliche Hilfen oder Bildungs- und Betreuungsangebote von Geburt an notwendig, um Auswege aus der Armut zu eröffnen.

Integration in Arbeit ist ein wichtiger Baustein der Armutsbekämpfung, greift aber bei denen zu kurz, die aufgrund von Alter, Gesundheitszustand oder Familiensituation nur schwer oder eingeschränkt in Arbeit vermittelt werden können. $\mathrm{Zu}-$ dem würde ein solcher Ansatz voraussetzen, dass tatsächlich genügend Arbeitsplätze zu ausreichenden Löhnen für alle Arbeitssuchenden zur Verfügung stehen. Das ist in Deutschland nicht der Fall.

Armut wird versucht, über das zur Verfügung stehende Einkommen festzulegen. Je nach Berechnungsgrundlage gibt es hierzu differierende Zahlen (vgl. Tabelle S. 131). Die Berechnungen des Deutschen Instituts für Wirtschaft (DIW) vom Februar 2010 gehen von 11,5 Millionen armen Menschen aus. Dies entspricht einer Armutsquote von 14 Prozent. Rund 6,8 Millionen Menschen erhielten im Juni 2009 Arbeitslosengeld II oder Sozialgeld. 
Die Nationale Armutskonferenz

Die Nationale Armutskonferenz gründete sich im Herbst 1991 als deutsche Sektion des Europäischen Armutsnetzwerks. Ziel der Zusammenarbeit ist es, Armut zu überwinden und die Selbsthilfeansätze der von Armut betroffenen oder bedrohten Menschen zu unterstützen. Sie sieht ihren Auftrag unter anderem darin, einen Beitrag zu einer veränderten Politik zu leisten, damit die Le-
Die Nationale Armutskonferenz besteht aus folgenden Mitgliedern: Arbeiterwohlfahrt Bundesverband, Armut und Gesundheit in Deutschland, BAG Prekäre Lebenslagen, BAG Schuldnerberatung, BAG Soziale Stadtentwicklung und Gemeinwesenarbeit, BAG Wohnungslosenhilfe, Bundesverband Die Tafeln, Der Paritätische Gesamtverband, Deutscher Bundesjugendring, Deutscher Caritasverband, Deutscher Gewerkschaftsbund, Deutsches Rotes Kreuz, Diakonisches

\section{"Wollen wir soziale Ungleichbeit vermindern, müssen wir mehr in Gerechtigkeit investieren "}

benslage armer Menschen verbessert und strukturelle Überwindung von Armutsbedrohung erreicht wird. Das Gespräch mit von Armut betroffenen Menschen ist erstes Anliegen der Nationalen Armutskonferenz.
Werk der Evangelischen Kirche in Deutschland, Zentralwohlfahrtsstelle der Juden in Deutschland. Sprecher der Nationalen Armutskonferenz ist Pfarrer Dr. Wolfgang Gern, der Vorstandsvorsitzende des Diakonischen Werks in Hessen und Nassau. Stellvertretende Sprecherin ist Michaela Hofmann, Referentin für Allgemeine Sozialberatung, Armutsfragen, Frauenhäuser und Gewaltschutz im Diözesan-Caritasverband Köln.

\section{Europäisches Jahr zur Bekämpfung von Armut und sozialer Ausgrenzung 2010}

Das Europäische Parlament und der Europäische Rat haben am 22. Oktober 2008 das Jahr 2010 zum »Europäischen Jahr zur Bekämpfung von Armut und sozialer Ausgrenzung « ausgerufen. Im Beschluss der Europäischen Union heißt es dazu wörtlich: »Die soziale Ausgrenzung steht dem Wohlergehen der Bürgerinnen und Bürger entgegen, hindert sie daran, sich zu äußern und an der Gesellschaft teilzuhaben. Dieser Aspekt sollte daher im Europäischen Jahr zur Bekämpfung von Armut und sozialer Ausgrenzung ... entsprechend herausgestellt werden. Das Europäische Jahr sollte aktive Eingliederungsstrategien als Mittel zur Verhinderung von Armut und sozialer Ausgrenzung fördern und im Rahmen der offenen
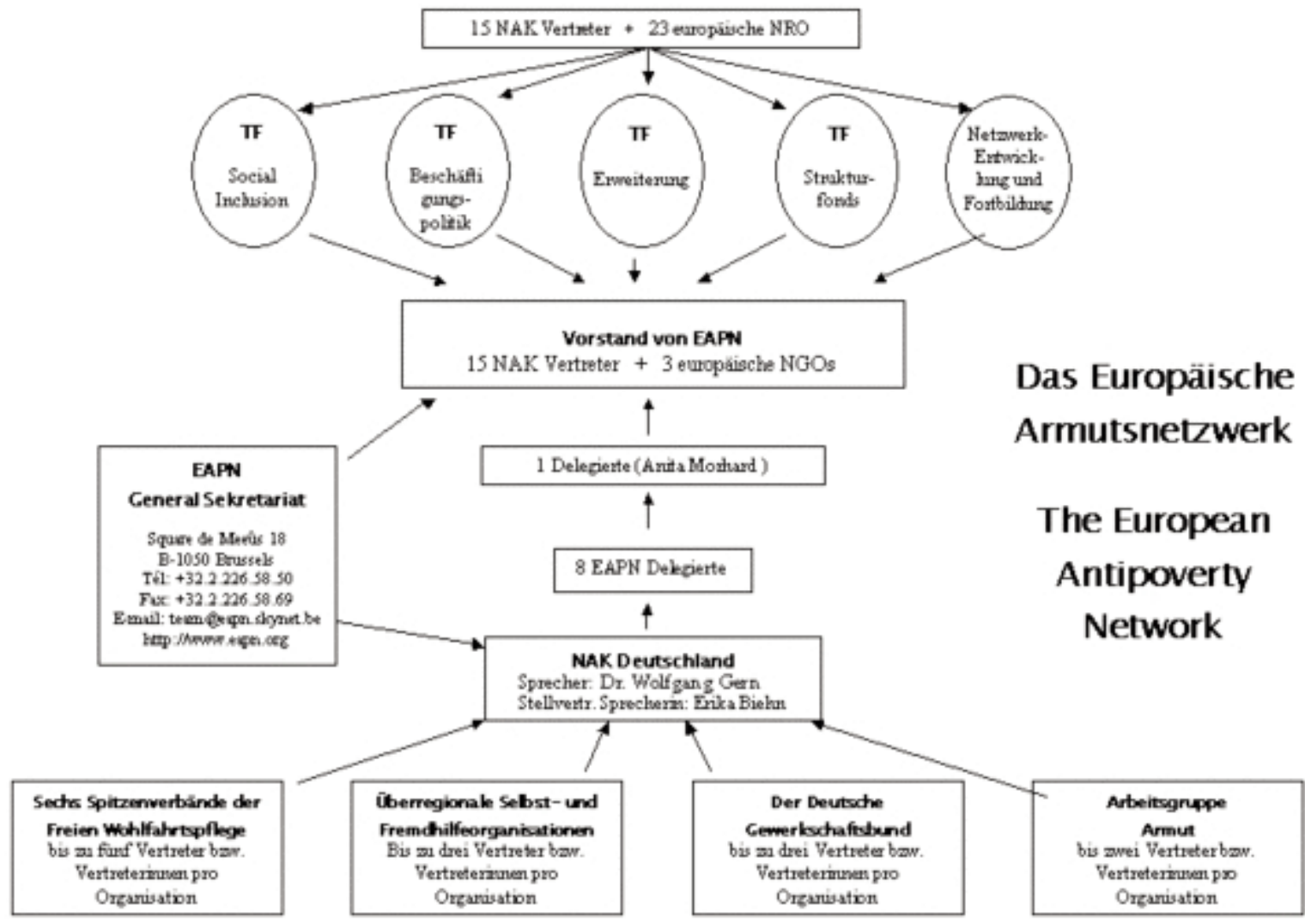

Die Nationale Armutskonferenz in Deutschland ist in das European Antipoverty Network (EAPN) eingebunden. Internet http://www.eapn.org 


\begin{tabular}{|l|l|l|}
\hline Berechnungsgrundlage & $\begin{array}{l}\text { Armutsschwelle für } \\
\text { Alleinstehende }\end{array}$ & $\begin{array}{l}\text { Armutsquote der } \\
\text { Bevölkerung }\end{array}$ \\
\hline $\begin{array}{l}\text { EU-einheitliches Verfahren nach } \\
\text { den Laeken-Indikatoren }\end{array}$ & 781 Euro im Monat & $13 \%$ \\
\hline Sozioökonomisches Panel & 880 Euro im Monat & $18 \%$ \\
\hline $\begin{array}{l}\text { Einkommens- und Verbrauchs- } \\
\text { stichprobe }\end{array}$ & 980 Euro im Monat & \\
\hline Steuerliches Existenzminimum & 8.004 Euro im Jahr & \\
\hline Pfändungsfreigrenze & 989,99 Euro im Monat & \\
\hline
\end{tabular}

Die Armutsschwelle in Deutschland variiert je nach der Berechnungsgrundlage.

Methode der Koordinierung zur Verbreitung bewährter Vorgehensweisen auf diesem Gebiet beitragen. « (Quelle: Beschluss der Europäischen Union, 22.10.2008)

In Deutschland ist das Bundesministerium für Arbeit und Soziales mit der Durchführung des Europäischen Jahres zur Bekämpfung von Armut und sozialer Ausgrenzung beauftragt worden. Es sollen drei große Themenfelder sichtbar werden: "Jedes Kind ist wichtig - Entwicklungschancen verbessern! « » Wo ist der Einstieg? - Mit Arbeit Hilfebedürftigkeit überwinden! « »Integration statt Aus- grenzung - Selbstbestimmte Teilhabe für alle Menschen! «

Das Bundesministerium für Arbeit und Soziales beteiligt als Nationale Durchführungsstelle die Akteure im Einsatz gegen Armut und soziale Ausgrenzung auf nationaler, regionaler und lokaler Ebene bei der Planung und Durchführung des Jahres. Ein besonderes Augenmerk im Europäischen Jahr zur Bekämpfung von Armut und sozialer Ausgrenzung liegt auf der Teilhabe der von Armut und Ausgrenzung betroffenen Bürgerinnen und Bürger.

\section{Lissabon-Strategie}

Obwohl die Europäische Union einige der höchst entwickelten Sozialschutzsysteme der Welt hat, sind noch immer etwa 80 Millionen ihrer Bürgerinnen und Bürger von Armut bedroht. Um dem entgegenzuwirken, verpflichteten sich die EU-Chefs im Rahmen der Lissabon-Strategie, Armut zu bekämpfen, und vereinbarten ein koordiniertes Vorgehen im Kampf gegen Ausgrenzung, das auch gemeinsame Ziele umfasst. Daraufhin verabschiedeten die Mitgliedstaaten nationale Aktionspläne (jetzt nationale Strategieberichte), die darauf abzielten, durch eine Verstärkung nationaler, regionaler und kommunaler Maßnahmen, eine bessere Koordinierung der gesamten politischen Bemühungen und die Einbindung aller einschlägigen Akteure gegen Armut und Ausgrenzung anzugehen. Die Bekämpfung von Armut und sozialer Ausgrenzung geht Hand in Hand mit anderen Zielen von Lissabon, insbesondere mit der Förderung der Beschäftigung und des Wirtschaftswachstums.

\title{
Akzeptanz von Hartz IV?
}

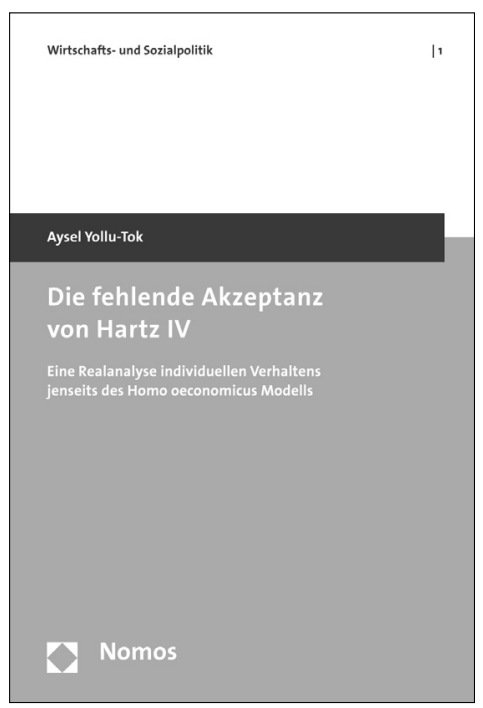

\author{
Die fehlende Akzeptanz von Hartz IV \\ Eine Realanalyse individuellen Verhaltens jenseits des \\ Homo oeconomicus Modells \\ Von Dr. Aysel Yollu-Tok \\ 2010, 216 S., brosch., 29,- $€$ ISBN 978-3-8329-5061-3 \\ (Wirtschafts- und Sozialpolitik, Bd. 1)
}

Die vorliegende Publikation geht der Frage nach, auf welche Verhaltensmotive die Akzeptanzprobleme der Hartz IV-Reform zurückgeführt werden können. Hierzu wird ein neues Verhaltensmodell entwickelt, womit Fragen wie der Einfluss von eigennützigen Präferenzen, von Gerechtigkeitsvorstellungen sowie Vorstellungen zu Exklusion und Inklusion geklärt werden können.

Bitte bestellen Sie im Buchhandel oder

versandkostenfrei unter $\checkmark$ www.nomos-shop.de

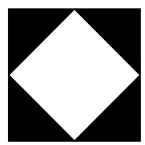

Nomos 